\title{
Estimulação transcraniana não invasiva em indivíduos com paralisia cerebral: uma revisão integrativa da literatura
}

\author{
Noninvasive brain stimulation in subjects with cerebral palsy: a integrative literature review
}

\author{
Aline Freitas Gomesa, Juliana Saibt Martins ${ }^{b}$ \\ a Acadêmica de Fisioterapia. Centro Universitário Franciscano, Santa Maria, RS \\ b Fisioterapeuta. Doutora em Ciências Biológicas (Bioquímica) pela Universidade Federal do Rio Grande do Sul. \\ Docente do Curso de Fisioterapia do Centro Universitário Franciscano, Santa Maria, RS.
}

Objetivo: Verificar na literatura a finalidade do uso da estimulação transcraniana não invasiva nos indivíduos com paralisia cerebral (PC).

Materiais e Métodos: Este estudo se caracteriza como uma revisão integrativa da literatura. A coleta dos dados foi realizada de agosto a setembro de 2015, utilizando as bases eletrônicas Scielo, Medline/PubMed, PEDro e Lilacs. Os descritores e operadores boleanos utilizados foram congenital hemiplegia OR cerebral palsy AND transcranial magnetic stimulation $O R$ transcranial direct current stimulation. Foram incluídos na amostra artigos publicados entre os anos de 2005 e 2015, nos idiomas inglês, espanhol e português.

Resultados: A busca nas bases de dados resultou na coleta de 214 artigos, dos quais 12 preencheram os critérios de inclusão. O maior número de publicações que compuseram a amostra foi publicado entre os anos de 2013 e 2015. Oito estudos aplicaram a estimulação magnética transcraniana (EMT) para realização de diagnóstico, mapeamento cortical e avaliação da função da mão e quatro utilizaram a estimulação por corrente contínua (ETCC) e verificaram seu efeito sobre a distonia e a marcha de indivíduos com PC. Em relação ao tratamento de indivíduos com PC, os estudos mostraram resultados importantes do uso de estimulação transcraniana, sobretudo quando associado à fisioterapia.

Conclusão: A pesquisa mostrou que a estimulação transcraniana não invasiva, por meio da EMT e da ETCC, tem sido utilizada na PC com a finalidade de tratamento e diagnóstico.

Palavras-chave: estimulação magnética transcraniana; estimulação transcraniana por corrente contínua; paralisia cerebral.

\section{ABSTRACT}

\begin{abstract}
Objective: To verify in the literature the purpose of using non-invasive transcranial magnetic stimulation in individuals with cerebral palsy $(C P)$.

Materials and Methods: This study is an integrative review of literature. The data collection was conducted from August to September, 2015, using the electronic data bases Scielo, Medline/PubMed, PEDro and Lilacs. The descriptors and Boolean operators used were congenital hemiplegia OR cerebral palsy AND transcranial magnetic stimulation OR transcranial direct current stimulation. The sample included papers published between 2005 and 2015, written in English, Spanish and Portuguese.

Results: The research on the data bases resulted in 214 papers, from which 12 fulfilled the established criteria. The highest number of publications that composed the sample was published between the years of 2013 and 2015. Eight (8) studies used transcranial magnetic stimulation (TMS) for carrying out the diagnosis, cortical mapping, and evaluating hand function, and four (4) used transcranial direct current stimulation (TDCS) and verified its effects on dystonia and gait of individuals with CP. In relation to the treatment of individuals with CP, studies showed important results in the use of transcranial stimulation, especially when associated with physiotherapy.
\end{abstract}

Conclusion: This study showed that non-invasive transcranial stimulation, through TMS and TDCS has been used in CP with the purpose of treating and diagnosing.

Keywords: transcranial magnetic stimulation; transcranial direct current stimulation; cerebral palsy.

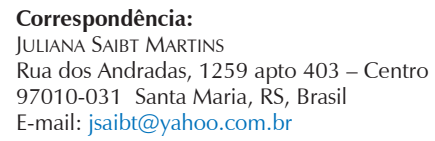




\section{INTRODUÇÃO}

A paralisia cerebral (PC) ou encefalopatia crônica não progressiva da infância, é definida como um grupo de desordens do movimento, tônus e de postura consequentes a lesões que ocorrem no sistema nervoso central (SNC) em fase de maturação $0^{1,2,3}$. Entre todas as deficiências que acarretam incapacidade motora em crianças, a PC apresenta a maior incidência. Acredita-se que as inadequadas condições de assistência à saúde no período pré e perinatal possam contribuir significativamente para esse panorama ${ }^{4,5}$.

A PC pode manifestar-se por variadas formas clínicas e sua classificação é realizada de acordo com a extensão do distúrbio motor, a intensidade e sua caracterização semiológica ${ }^{6}$, entre outros. O grau de limitação dos indivíduos acometidos varia de acordo com a área e extensão da lesão ${ }^{7,8}$. Nesse sentido, apesar da principal disfunção ser de ordem motora, o quadro clínico frequentemente está associado a distúrbios sensoriais, déficit intelectual e epilepsia ${ }^{8,9,10}$. Assim, o tratamento da PC deve envolver uma equipe multidisciplinar.

Embora o prejuízo sensório-motor seja permanente nos indivíduos com PC, muitos recursos podem permitir que eles venham a desenvolver seu potencial máximo ${ }^{11}$. Dessa forma, a fisioterapia deve iniciar precocemente e deve ser contínua, e ter como principais objetivos a inibição da atividade reflexa anormal e a melhora da capacidade funcional, visando maior independência dessa população ${ }^{12}$.

Os treinos funcionais e a estimulação sensorial são importantes recursos fisioterapêuticos que promovem a aprendizagem motora ${ }^{13,14}$. Eles promovem ativação do córtex primário e algum grau de reorganização cortical. No entanto, podem não ser suficientes para estimular todo o potencial de plasticidade do sistema nervoso humano ${ }^{15}$. Nesse contexto, a estimulação transcraniana não invasiva é um método promissor e inovador que pode intervir no funcionamento do cérebro. Seus benefícios se dão pela capacidade de modulação da função cerebral e podem promover excitação ou inibição de áreas específicas. As duas principais técnicas de estimulação cerebral não invasiva são a estimulação magnética transcraniana (EMT) e a estimulação por corrente contínua (ETCC). Ambas atingem diretamente as estruturas cerebrais, promovendo alterações plásticas de maneira focal, indolor e com largo limite de segurança ${ }^{16,17}$.

Estudos mostram que a estimulação cerebral é um importante recurso para o diagnóstico, monitoramento e tratamento de doenças neurológicas e psiquiátricas, com inúmeros benefícios já reconhecidos ${ }^{18}$. A pesquisa nessa área vem sendo intensificada no intuito de estabelecer consensos e protocolos de segurança para sua aplicação em outras disfunções ${ }^{18}$. O presente estudo teve por objetivo investigar a finalidade do uso da estimulação transcraniana não invasiva em indivíduos com PC.

\section{MATERIAIS E MÉTODOS}

Este estudo caracteriza-se como exploratório, do tipo revisão integrativa da literatura. A busca dos estudos ocorreu nos meses de agosto e setembro de 2015, nas bases de dados eletrônicas Lilacs (Literatura Latino-Americana em Ciências da Saúde), Medline (Medical Literature Analysis and Retrieval System Online), Pubmed (National Library of Medicine), PEDro (Physiotherapy evidence database), e Scielo (Scientific Electronic Library Online). Os descritores e operadores boleanos utilizados foram: hemiplegia/hemiplegia/hemiplejía $O R$ paralisia cerebral/cerebral palsy AND estimulação magnética transcraniana/transcranial magnetic stimulation/estimulación magnética transcraneal $O R$ estimulação transcraniana por corrente contínua/transcranial direct current stimulation/ estimulación transcraneal de corriente directa.

Os critérios de inclusão utilizados para selecionar os artigos foram: artigos completos e disponíveis online na íntegra, no período de 2005 a 2015, publicados nos idiomas português, inglês e espanhol, que abordassem a relação entre o uso da estimulação transcraniana não invasiva e paralisia cerebral. Estudos de revisão bibliográfica e os artigos em duplicata ou triplicata foram excluídos da amostra.

Após a busca nas bases de dados, foi realizada a avaliação dos títulos e dos resumos (abstracts) e os estudos que contemplaram os critérios de inclusão foram selecionados para leitura na íntegra. Foram registradas as seguintes características das publicações: nome do primeiro autor e ano de publicação, objetivos dos estudos, amostra, tipo de estimulação, além da metodologia e finalidade da aplicação da estimulação.

\section{RESULTADOS E DISCUSSÃO}

A busca nas bases de dados resultou na coleta de 214 artigos, dos quais 12 preencheram adequadamente os critérios e foram selecionados para inclusão no estudo. A Figura 1 apresenta o fluxograma com a estratégia de seleção dos artigos incluídos. O maior número de publicações que compuseram a amostra do presente estudo foi publicado entre os anos de 2013 e 2015.

Este estudo mostrou que a EMT e a ETCC são as principais técnicas de estimulação transcraniana não invasiva utilizadas atualmente na PC. As duas modalidades se constituem intervenções neuromoduladoras que induzem alterações na excitabilidade do córtex motor humano ${ }^{20,21}$. Constatou-se que, para a realização de oito estudos que compuseram a amostra, foi aplicada a EMT e em quatro, a ETCC. 


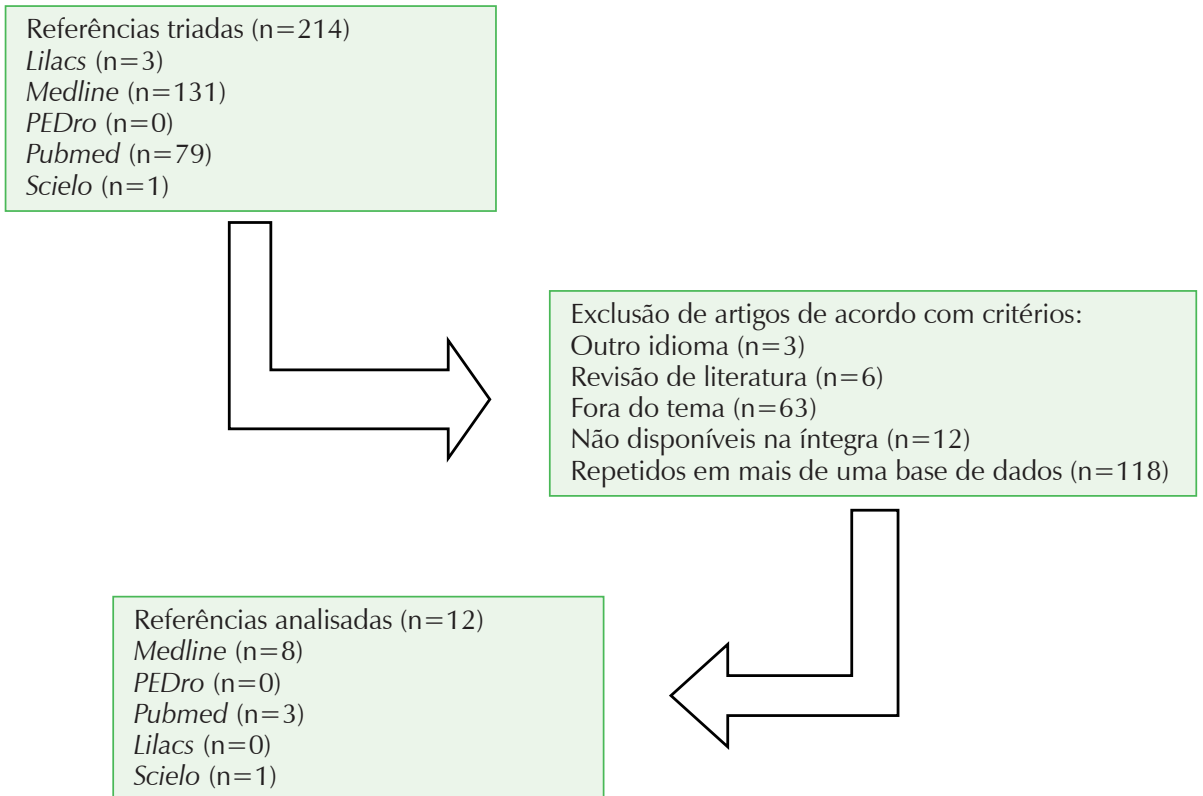

Figura 1. Fluxograma com a estratégia de seleção dos artigos.

A principal diferença entre as estimulações supracitadas é que na ETCC não ocorre a despolarização do neurônio, diferentemente do que ocorre na EMT. Além disso a ETCC estimula o córtex cerebral por meio de eletrodos de superfície, e seus efeitos são alcançados a partir do movimento de cargas elétricas entre os eletrodos, sendo que o polo do ânodo (positivo) aumenta a excitabilidade neuronal e o polo do cátodo (negativo) (reduz a excitabilidade)22,23. Já a aplicação da EMT ocorre por intermédio de uma bobina que gera um campo elétrico, que pode ser regulado de acordo com efeito desejado, ou seja, altas frequências $(5,10,20 \mathrm{~Hz})$ aumentam a atividade cortical e frequências baixas (0.5 a $1 \mathrm{~Hz}$ ) diminuem essa atividade.

Existem três formas de aplicar a EMT: pulso simples, utilizado para realizar mapeamento cerebral; pulso pareado, para avaliações neurofisiológicas e pulso repetitivo, para o tratamento de doenças neuropsiquiátricas ${ }^{24,25,26}$. Outra diferença entre as modalidades aplicadas na PC é que a ETCC apresenta vantagens quando comparada à EMT quanto à sua portabilidade e baixo custo ${ }^{27}$.

Quanto às aplicações dessas técnicas em indivíduos com PC, constatou-se, como o presente estudo mostrou que atualmente a ETCC é usada com foco no tratamento, e a EMT no diagnóstico, por meio de mapeamento do córtex cerebral. As quatro pesquisas que abordaram a utilização da ETCC verificaram seu efeito sobre a distonia e a marcha de indivíduos com PC. Nos estudos sobre distonia, foi aplicada corrente catódica a fim de desencadear uma inibição, visto que o distúrbio é causado pela excitabilidade do córtex motor. O estudo que aplicou apenas duas sessões obteve um efeito reduzido sobre os movimentos involuntários nos indivíduos com PC, os quais possuíam idades entre sete e 19 anos $^{28}$. Em busca de mais respostas, o mesmo grupo de pesquisadores realizou novo estudo baseando-se nos anteriores, dessa vez em amostra de nove indivíduos com distonia primária ou secundária, com idade entre dez e 21 anos. Eles aplicaram maior quantidade de sessões com alternação da estimulação anódica com a catódica. No entanto os resultados não apontaram que esta seja uma técnica clinicamente viável para o tratamento da distonia da infância ${ }^{29}$. De fato, a distonia é um distúrbio crônico do movimento, com difícil diagnóstico e previsão prognóstica, além de ter causa desconhecida, o que dificulta ainda mais seu tratamento ${ }^{30}$. Caracterizada por contrações musculares involuntárias e contínuas, pode afetar qualquer parte do corpo e causar prejuízos funcionais ${ }^{31}$.

Quanto ao uso da ETCC na marcha de crianças com PC, os estudos analisados associaram a estimulação por corrente anódica com terapia física. Os autores concluíram que as alterações neuroplásticas causadas pela ETCC são potencializadas quando esse recurso é associado com terapia física ${ }^{32,33}$. De fato, a literatura evidencia que os mecanismos do exercício que geram benefícios no cérebro possivelmente estão associados a mudanças na plasticidade sináptica, contribuindo para a função cerebral ${ }^{34}$. A repetição dos exercícios é um princípio importante para facilitar a neuroplasticidade. Várias técnicas de reabilitação usam como elemento central a repetição de um conjunto de 
Tabela 1. Publicações que compuseram a amostra do estudo.

\begin{tabular}{|c|c|c|c|c|c|}
\hline Autor/Ano & Objetivo do estudo & Amostra & $\begin{array}{c}\text { Tipo de } \\
\text { estimulação }\end{array}$ & Metodologia de aplicação & Finalidade da estimulação \\
\hline Young et al. ${ }^{28}$ & $\begin{array}{l}\text { Verificar se a estimulação catódica } \\
\text { por corrente contínua reduz a } \\
\text { excitabilidade do córtex motor de } \\
\text { crianças com distonia. }\end{array}$ & $\begin{array}{l}14 \text { indivíduos com distonia } \\
\text { primária ou secundária } \\
\text { afetando uma ou ambas as } \\
\text { mãos ( } 7-19 \text { anos). }\end{array}$ & ETCC & $\begin{array}{l}\text { A aplicação foi realizada em } \\
\text { duas visitas de aproximada- } \\
\text { mente duas horas. Em uma foi } \\
\text { aplicada estimulação catódica } \\
\text { real e na outra uma estimulação } \\
\text { placebo. O cátodo foi colocado } \\
\text { ao longo do córtex motor e o } \\
\text { ânodo contralateral ao cátodo. }\end{array}$ & $\begin{array}{l}\text { Tratamento: reduzir a } \\
\text { excitabilidade do córtex } \\
\text { motor para promover inibição } \\
\text { e reduzir sintomas distônicos. }\end{array}$ \\
\hline Bhanpuri et al. ${ }^{29}$ & $\begin{array}{l}\text { Investigar a hipótese de que a } \\
\text { neuromodulação pode reduzir a } \\
\text { atividade anormal do córtex } \\
\text { motor causada pela distonia. }\end{array}$ & $\begin{array}{l}\text { Nove indivíduos com distonia } \\
\text { primária ou secundária } \\
\text { (10-21 anos). }\end{array}$ & ETCC & $\begin{array}{l}\text { Nove indivíduos participaram } \\
\text { de dois experimentos: uma } \\
\text { sessão diária com duração de } \\
\text { uma hora a uma hora e meia, } \\
\text { por duas semanas consecutivas, } \\
\text { o que totalizou } 10 \text { sessões de } \\
\text { ETCC catódica ou placebo. } \\
\text { Após duas semanas de intervalo } \\
\text { os pacientes receberam o } \\
\text { mesmo protocolo, porém com } \\
\text { ETCC anódica ou placebo. }\end{array}$ & $\begin{array}{l}\text { Tratamento: reduzir a } \\
\text { excitabilidade do córtex } \\
\text { motor para promover inibição } \\
\text { e reduzir sintomas distônicos. }\end{array}$ \\
\hline Grecco et al. ${ }^{32}$ & $\begin{array}{l}\text { Determinar o efeito de uma } \\
\text { única sessão de ETCC sobre } \\
\text { características da marcha. }\end{array}$ & $\begin{array}{l}25 \text { crianças espásticas } \\
\text { (6-10 anos). }\end{array}$ & ETCC & $\begin{array}{l}\text { Uma única sessão de ETCC } \\
\text { anódica aplicada no córtex } \\
\text { motor primário durante } 20 \\
\text { minutos. Os participantes foram } \\
\text { divididos em dois grupos, } \\
\text { experimental ou controle. }\end{array}$ & $\begin{array}{l}\text { Tratamento: potencializar } \\
\text { padrões motores através do } \\
\text { aumento da excitabilidade do } \\
\text { córtex motor. }\end{array}$ \\
\hline Grecco et al. ${ }^{33}$ & $\begin{array}{l}\text { Comparar o efeito da ETCC } \\
\text { anódica sobre o treino de marcha } \\
\text { na esteira. Determinar se os } \\
\text { efeitos se mantinham após } 1 \text { mês } \\
\text { de intervenção. }\end{array}$ & $\begin{array}{l}39 \text { crianças espásticas } \\
\text { (5-10 anos) }\end{array}$ & ETCC & $\begin{array}{l}\text { As crianças foram alocadas } \\
\text { aleatoriamente para o grupo } \\
\text { controle ou experimental. } \\
\text { Foram realizadas } 10 \text { sessões } \\
\text { (cinco vezes na semana por } \\
\text { duas semanas consecutivas) de } \\
\text { ETCC anódica, por } 20 \text { minutos } \\
\text { com a criança reali- } \\
\text { zando treinamento em esteira. }\end{array}$ & $\begin{array}{l}\text { Tratamento: promover } \\
\text { estimulação do córtex motor, } \\
\text { levar a um aumento sináptico } \\
\text { local e, como consequência, } \\
\text { alterar o padrão de } \\
\text { plasticidade mal adaptativa } \\
\text { que surge após a lesão. }\end{array}$ \\
\hline Niek e Van Der et al. ${ }^{42}$ & $\begin{array}{l}\text { Estudar o potencial da } \\
\text { neuroimagem neonatal para } \\
\text { predizer o resultado motor e } \\
\text { determinar o padrão de inervação } \\
\text { motora. }\end{array}$ & $\begin{array}{l}37 \text { indivíduos hemiparéticos } \\
\text { (7-18 anos) }\end{array}$ & EMT & $\begin{array}{l}\text { Bobina em forma de "oito"; } \\
\text { eletrodos localizados nos córtex } \\
\text { motor direito e esquerdo. }\end{array}$ & $\begin{array}{l}\text { Diagnóstica/ mapeamento } \\
\text { cortical: determinar o tipo de } \\
\text { organização motora cortical } \\
\text { (normal, mista, ipsilateral). }\end{array}$ \\
\hline Holmstrom et al. ${ }^{43}$ & $\begin{array}{l}\text { Investigar as relações entre } \\
\text { a função da mão, as lesões } \\
\text { cerebrais e os padrões de } \\
\text { projeção motora na PC. }\end{array}$ & 17 indivíduos (7-16 anos). & EMT & $\begin{array}{l}\text { Bobina em forma de "oito"; } \\
\text { pulso único. }\end{array}$ & $\begin{array}{l}\text { Diagnóstico e mapeamento } \\
\text { cortical; com IRM determinar } \\
\text { o tipo, local e extensão } \\
\text { da lesão. Com EMT obter } \\
\text { informações sobre a } \\
\text { organização das projeções } \\
\text { corticomotoras. }\end{array}$ \\
\hline Mackey et al. ${ }^{44}$ & $\begin{array}{l}\text { Relacionar a reorganização do } \\
\text { córtex motor, tipo de lesão e } \\
\text { função do membro superior. }\end{array}$ & $\begin{array}{l}20 \text { indivíduos hemiparéticos } \\
\text { (12-25 anos) }\end{array}$ & EMT & $\begin{array}{l}\text { Bobina em forma de "oito"; } \\
\text { pulso único. }\end{array}$ & $\begin{array}{l}\text { Diagnóstico e mapeamento } \\
\text { cortical: avaliar a integridade } \\
\text { funcional e organização das } \\
\text { vias motoras corticais. }\end{array}$ \\
\hline Islam et al. ${ }^{45}$ & $\begin{array}{l}\text { Explorar variações individuais no } \\
\text { resultado da função da mão após } \\
\text { terapia por contensão induzida } \\
\text { (TCl). }\end{array}$ & $\begin{array}{l}26 \text { indivíduos hemiparéticos } \\
\text { (8-18 anos). }\end{array}$ & EMT & $\begin{array}{l}\text { Bobina em forma de "oito"; } \\
\text { pulso único. }\end{array}$ & $\begin{array}{l}\text { Diagnóstico e mapeamento } \\
\text { cortical: determinar o tipo de } \\
\text { organização das projeções } \\
\text { corticomotoras. }\end{array}$ \\
\hline Pihko et al. ${ }^{46}$ & $\begin{array}{l}\text { Investigar se as alterações no } \\
\text { córtex motor após lesão cerebral } \\
\text { precoce afetaria o padrão de } \\
\text { reatividade das oscilações } \\
\text { corticais sensório-motoras em } \\
\text { crianças com PC. }\end{array}$ & $\begin{array}{l}12 \text { indivíduos hemiplégicos } \\
\text { (11-17 anos) }\end{array}$ & EMT & $\begin{array}{l}\text { Bobina em forma de "oito"; } \\
\text { Pulsos bifásicos individuais }\end{array}$ & $\begin{array}{l}\text { Diagnóstico e mapeamento } \\
\text { cortical: identificar as } \\
\text { representações corticais dos } \\
\text { músculos intrínsecos da mão. }\end{array}$ \\
\hline Koerte et al. ${ }^{47}$ & $\begin{array}{l}\text { Caracterizar os déficits } \\
\text { motores de crianças com PC } \\
\text { e leucomalácia periventricular } \\
\text { quanto à integridade da } \\
\text { substância branca e função } \\
\text { motora da mão. }\end{array}$ & $\begin{array}{l}\text { Sete indivíduos quadriplégicos } \\
\text { (idade média } 18 \text { anos) }\end{array}$ & EMT & Bobina em forma de oito. & $\begin{array}{l}\text { Diagnóstico e mapeamento } \\
\text { cortical: identificar as } \\
\text { representações corticais dos } \\
\text { músculos intrínsecos da mão } \\
\text { e os parâmetros de inibição } \\
\text { inter-hemisférica. }\end{array}$ \\
\hline Kesar et al. ${ }^{48}$ & $\begin{array}{l}\text { Investigar os padrões de reorga- } \\
\text { nização motora cortical e sua } \\
\text { relação com a função motora. }\end{array}$ & $\begin{array}{l}13 \text { indivíduos hemi e } \\
\text { diparéticos } \\
\text { (9-16 anos) }\end{array}$ & EMT & Bobina circular. & $\begin{array}{l}\text { Diagnóstico e mapeamento } \\
\text { cortical: identificar a topografia } \\
\text { dos mapas corticais motores. }\end{array}$ \\
\hline Bleyenheuft et al. ${ }^{49}$ & $\begin{array}{l}\text { Registrar as alterações neurofisio- } \\
\text { siológicas antes e após tratamento } \\
\text { bimanual e documentar a } \\
\text { relação de complementaridade } \\
\text { entre a EMT, IRM e ITD, bem } \\
\text { como seus possíveis efeitos. }\end{array}$ & $\begin{array}{l}\text { Duas crianças hemiparéticas } \\
\text { (6 e } 9 \text { anos). }\end{array}$ & EMT & $\begin{array}{l}\text { Bobina em forma de oito; pulso } \\
\text { único. A EMT foi realizada } \\
\text { antes e após } 90 \text { horas de } \\
\text { tratamento bimanual intensivo, } \\
\text { sendo movida ao longo da } \\
\text { cabeça. }\end{array}$ & $\begin{array}{l}\text { Diagnóstico e mapeamento } \\
\text { cortical: identificar a } \\
\text { topografia dos mapas corticais } \\
\text { motores e sua excitabilidade. }\end{array}$ \\
\hline
\end{tabular}

EMT: estimulação magnética transcraniana; ETCC: estimulação transcraniana por corrente contínua; PC: paralisia cerebral; IRM: imagem por ressonância magnética; ITD: imagem por tensor de difusão. 
exercícios para promover recuperação do $\mathrm{SNC}^{41}$. Nesse aspecto, a fisioterapia dispõe de técnicas como o Conceito Bobath $^{35}$, a hidroterapia ${ }^{36}$, a terapia por contensão induzida $(\mathrm{TCl})^{37}$, a realidade virtual $(\mathrm{RV})^{38}$, a facilitação neuromuscular proprioceptiva $(\mathrm{FNP})^{39}$, as quais possibilitam, por meio da experiência, a adaptação das estruturas e a melhora da função do sistema nervoso ${ }^{40}$. Os estudos incluídos na amostra mostraram resultados importantes do uso da estimulação transcraniana em indivíduos com PC, sobretudo quando associado com a fisioterapia.

Os resultados de uma pesquisa aplicada em 25 crianças com PC espástica, de seis a dez anos, que utilizou uma única sessão de ETCC por 20 minutos mostraram redução das oscilações e aumento da velocidade, comprimento do passo e da passada, resultados que não se mantiveram por mais de 20 minutos após estimulação ${ }^{32}$. Com intuito de verificar se a aplicação com maior número de sessões manteria os efeitos por um período mais longo, realizou-se nova pesquisa com dez sessões de ETCC anódica durante o treino em esteira. Nesse estudo a amostra foi composta por 39 crianças, com idades entre cinco a dez anos, com PC espástica, que haviam alcançado a marcha independente há, pelo menos, um ano. Obteve-se uma melhora na marcha e os efeitos foram mantidos após um mês do protocolo de reabilitação ${ }^{33}$.

Observou-se nesse estudo, que a ETCC tem sido aplicada para tratamento, porém as publicações mostraram que seus efeitos em indivíduos com PC são ainda limitados e atribuídos principalmente à melhora da marcha.

Por outro lado, a EMT tem sido utilizada na PC para realizar mapeamento cerebral, determinando o tipo/padrão de organização cortical. A EMT de pulso simples, em associação com a imagem por ressonância magnética (IRM), foi empregada na análise do mapeamento cerebral e avaliação da função da mão de indivíduos com PC unilateral ${ }^{42,43,44,45,46}$. A EMT parece mostrar-se um recurso importante para obter um prognóstico precoce nessa população ${ }^{44,45}$. De fato, PIHKO et al. ${ }^{46}$ ressaltam que o conhecimento preciso da organização da rede sensório-motora funcional pode ser útil para adaptar um tratamento individualizado para indivíduos com PC. Koerte $^{47}$ ressalta que a avaliação realizada pela EMT em conjunto com ressonância magnética oferece uma nova visão sobre a interação das estruturas neuroanatômicas centrais e seu impacto na função motora em crianças com PC espástica bilateral e leucomalácia periventricular. Outros dois estudos que compuseram a amostra utilizaram a EMT para investigar medidas neurofisiológicas e padrão cortical motor desencadeadas pelo tratamento intensivo e sua relação com a função motora ${ }^{48,49}$. Pesquisa recente, realizada com duas crianças hemiparéticas, de seis e nove anos, revelou que as alterações corticais que estão ligadas ao melhor desempenho motor podem ser desencadeadas por sessões de terapia intensiva e podem ser monitoradas por mapeamento cortical ${ }^{49}$. Nesse sentido, estudo confirma a importância da EMT para mapeamento do córtex motor para obter informações a respeito da integridade da via motora e sobre seu perfil de reorganização ${ }^{50}$. Kesar et al. ${ }^{48}$, em pesquisa com treze indivíduos espásticos com idades entre nove e 16 anos, encontraram resultados promissores utilizando EMT para definir a relação entre as alterações no mapa motor e deficiências funcionais, através da investigação dos padrões corticais motores em crianças com diplegia e hemiplegia e a relação entre a geometria córtico-motora e a função motora.

No presente estudo, as publicações mostraram resultados promissores da aplicação da estimulação transcraniana não invasiva na PC. No entanto, apesar de tratar-se de um método não invasivo, praticamente indolor e não acarretar efeitos colaterais ${ }^{51}$, é uma tecnologia relativamente recente e ainda não acessível a diversos centros de tratamento no país.

Embora os achados sejam relevantes e tenham permitido que o objetivo deste estudo fosse alcançado, cabe ressaltar que o recorte temporal para a busca dos artigos, de 2005 a 2015, bem como a não inclusão de estudos que não estavam disponíveis na íntegra on line constituem limitações importantes desta revisão.

\section{CONSIDERAÇÕES FINAIS}

A pesquisa mostrou que a estimulação transcraniana não invasiva, por meio da estimulação magnética transcraniana (EMT) e da estimulação transcraniana por corrente contínua (ETCC), tem sido utilizada na PC com a finalidade de tratamento e diagnóstico.

\section{REFERÊNCIAS}

1. Mancini MC, Fiúza PM, Rebelo JM, Magalhães LC, Coelho ZAC, Paixão ML, Gontijo APB, Fonseca ST. Comparação do desempenho de atividades funcionais em crianças com desenvolvimento normal e crianças com paralisia cerebral. Arq Neuro-Psiquiatr. 2002;60(2B):446-52. http://dx.doi.org/10.1590/S0004-282X 2002000300020

2. Bax M, Goldstein M, Rosenbaum P, Leviton A, Paneth N, Dan B, Jacobsson B, Damiano D. Proposed definition and classification of cerebral palsy. Dev Med Child Neurol. 2005;47:571-6. http:// dx.doi.org/10.1017/S001216220500112X

3. Rocha AP, Afonso DV, Morais RS. Relação entre desempenho funcional de crianças com paralisia cerebral e qualidade de vida relacionada à saúde de seus cuidadores. Fisioter Pesq. 2008; 15(3):292-7.

4. Brasileiro IC, Moreira TMM, Jorge MSB, Queiroz MVO, Mont'Alverne DG. Atividade e participação de crianças com paralisia cerebral conforme a classificação internacional de funcionalidade, incapacidade e saúde. Rev Bras Enferm. 2009;62(4):503-11. http:// dx.doi.org/10.1590/S0034-71672009000400002 
5. Amaral PP, Mazzitelli C. Alterações ortopédicas em crianças com paralisia cerebral da clínica-escola de Fisioterapia da Universidade Metodista de São Paulo. Rev Neuroc. 2003;1(11):29-33.

6. Assis-Madeira EA, Carvalho SG. Paralisia cerebral e fatores de risco ao desenvolvimento motor: revisão teórica. Cad Pós-Grad Dist Desenv. 2009;9(1):142-63.

7. Mello R, Ichisato SMT, Marcon SS. Percepção da família quanto à doença e ao cuidado fisioterapêutico de pessoas com paralisia cerebral. Rev Bras Enferm. 2012;65(1):104-9. http://dx.doi. org/10.1590/S0034-71672012000100015

8. Cesa CC, Alves MES, Meireles LCF, Fante F, Manacero SA. Avaliação da capacidade funcional de crianças com paralisia cerebral. Rev. CEFAC. 2014;16(4):1266-72. http://dx.doi.org/10.1590/1982021620146513

9. Simões CC, Silva L, Santos MR, Misko MD, Bousso RS. A experiência dos pais no cuidado dos filhos com paralisia cerebral. Rev Eletr Enf. 2013;15(1):138-45. http://dx.doi.org/10.5216/ree.v15i1.13464

10. Ustad T, Sorsdahl AB, Ljunggren AE. Effects of intensive physiotherapy in infants newly diagnosed with cerebral palsy. Pediatr Phys Ther. 2009;21(2):140-8. http://dx.doi.org/10.1097/ PEP.0b013e3181a3429e

11. Maranhão MM. Tipo de atividade e relação interpessoal entre fisioterapeuta e criança com paralisia cerebral no contexto de intervenção fisioterapêutica. Dynamis. 2008;14(1):89-95

12. Leite JMRS, Prado GF. Paralisia cerebral aspectos fisioterapêuticos e clínicos. Rev Neuroc. 2004;12(1):41-5. http://dx.doi.org/10.4181/ rnc.2004.12.41

13. Miranda PC, Lomarev M, Hallett M. Modeling the current distribution during transcranial direct current stimulation. Clin Neurophysiol. 2006;117(7):1623-9. http://dx.doi.org/10.1016/j. clinph.2006.04.009

14. Grecco LA, Duarte NA, de Mendonça ME, Pasini H, Lima VL, Franco RC, Oliveira LV, Carvalho PT, Corrêa JC, Collange NZ, Sampaio LM, Galli M, Fregni F, Oliveira CS. Effect of transcranial direct current stimulation combined with gait and mobility training on functionality in children with cerebral palsy: study protocol for a double-blind randomized controlled clinical trial. BMC Pediatr. 2013;13(168): 1-11. http://dx.doi.org/10.1186/1471-2431-13-168

15. Ferrari EM, Toyoda MS, Faleiros L. Plasticidade neural: relações com o comportamento e abordagens experimentais. Psic Teor e Pesq. 2001;17(2):187-94. http://dx.doi.org/10.1590/S010237722001000200011

16. Moffa AH, Valiengo L, Shiozawa P, Brunoni AR. Novel neurotherapeutics in psychiatry: use and rationale of transcranial direct current stimulation in major depressive disorder. Rev Psiq Clín. 2014;41(1):15-20. http://dx.doi.org/10.1590/010160830004111520

17. Müller VT, Santos PP, Carnaval T, Gomes MM, Fregni F. O que é estimulação magnética transcraniana? Rev Bras Neurol. 2013;49(1):20-31.

18. Eldaief MC, Press DZ, Pascual-Leone A. Transcranial magnetic stimulation in neurology: a review of established and prospective applications. Neurol Clin Pract. 2013;3(6):519-26. http://dx.doi. org/10.1212/01.CPJ.0000436213.11132.8e

19. Flamand VH, Schneider C. Noninvasive and painless magnetic stimulation of nerves improved brain motor function and mobility in a cerebral palsy case. Arch Phys Med Rehabil. 2014;95:1984-9. http://dx.doi.org/10.1016/j.apmr.2014.05.014
20. Page SJ, Cunningham DA, Blazak B. It Takes Two: Noninvasive Brain Stimulation With Neurorehabilitation. Arch Phys Med Rehabil. 2015;96(4 Suppl 2):S89-93. http://dx.doi.org/10.1016/j. apmr.2014.09.019

21. Okano AH, Montenegro RY, Farinatti PTV, Li LM, Brunoni AR, Fontes EB. Estimulação cerebral na promoção da saúde e melhoria do desempenho físico. Rev Bras Educ Fis Esporte. 2013;27(2):315-32. http://dx.doi.org/10.1590/S1807-55092013005000009

22. Machado S, Velasques B, Cunha M, Basile L, Budde H, Cagy M, Piedade R, Ribeiro P. Aplicações terapêuticas da estimulação cerebral por corrente contínua na neuroreabilitação clínica. Rev Neurocienc. 2009;17(3):298-300.

23. Brunoni AR, Nitsche MA, Bolognini N, Bikson M, Wagner T, Merabet L, Edwards DJ, Valero-Cabre A, Rotenberg A, Pascual-Leone A, Ferrucci R, Priori A, Boggio PS, Fregnin F. Clinical research with transcranial direct current stimulation (tDCS): Challenges and future directions. Brain Stimul. 2012;5:175-95. http://dx.doi. org/10.1016/j.brs.2011.03.002

24. Boggio PS, Fregni F, Rigonatti SP, Marcolin MA, Silva MTA. Estimulação magnética transcraniana na neuropsicologia: novos horizontes em pesquisa sobre o cérebro. Rev Bras Psiquiatr. 2006;28(1):44-9. http://dx.doi.org/10.1590/S1516-44462006000100010

25. Araújo HA, Iglesio RF, Correia GSC, Fernandes DTRM, Galhardoni R, Marcolin MA, Teixeira MJ, Andrade DC. Estimulação magnética transcraniana e aplicabilidade clínica: perspectivas na conduta terapêutica neuropsiquiátrica/ Transcranial magnetic stimulation and clinical applicability: perspectives in neuropsychiatric therapeutics. Rev Med. 2011;90(1):3-14.

26. Müller VT, Santos PP, Carnaval T, Gomes MM, Fregni F. O que é estimulação magnética transcraniana? Rev Bras Neurol. 2013; 49(1):20-31.

27. Shiozawa P, Silva ME, Fregni F, Brunoni AR, Cordeiro Q. Estimulação craniana por corrente contínua (ETCC) no tratamento de distúrbios psiquiátricos: o que sabemos até agora? Arq Med Hosp Fac Cienc Med Santa Casa São Paulo. 2013;58:34-7.

28. Young SJ, Bertucco M, Sanger TD. Cathodal transcranial direct current stimulation in children with dystonia: a shamcontrolled study. J Child Neurol. 2014;29(2):232-9. http://dx.doi. org/10.1177/0883073813492385

29. Bhanpuri NH, Bertucco $M$, Young SJ, Lee AA, Sanger TD. Multiday transcranial direct current stimulation causes clinically insignificant changes in childhood dystonia: a pilot study. J Child Neurol. 2015;30(12):1604-15. http://dx.doi. org/10.1177/0883073815575369

30. Biase NG, Pontes AL, Junior VS, Vieira VP, Zambonato P, Yazaki RK. Difícil manejo do paciente com distonia segmentar respiratória. Rev Bras Otorrinolaringol. 2007;73(2):278-83. http://dx.doi. org/10.1590/S0034-72992007000200021

31. Dias FV, Hounie AG, Corrêa H, Teixeira AL. Distonia primária e transtorno obsessivo-compulsivo. J Bras Psiquiatr. 2007;56(1): 60-63. http://dx.doi.org/10.1590/S0047-20852007000100013

32. Grecco LAC, Duarte NAC, Zanon N, Galli M, Fregni F, Oliveira CS. Effect of a single session of transcranial direct-current stimulation on balance and spatiotemporal gait variables in children with cerebral palsy: a randomized sham-controlled study. Braz J Phys Ther. 2014;18(5):419-27. http://dx.doi.org/10.1590/bjpt-rbf.2014.0053

33. Grecco LAC, Duarte NAC, Mendonça ME, Cimolin V, Galli M, Fregni F, Oliveira CS. Transcranial direct current stimulation during treadmill 
trainingin children with cerebral palsy: a randomized controlled double-blind clinical trial. Res Dev Disabil. 2014;35(11):2840-8. http://dx.doi.org/10.1016/j.ridd.2014.07.030

34. Gonçalves M.P, Tomaz C, Sangoi C. Considerações sobre envelhecimento, memória e atividade física. $\mathrm{R}$ Bras $\mathrm{Ci}$ e Mov. 2006;14(1):101-8.

35. Knox V, Evans AL. Evaluation of the functional effects of a course of Bobath therapy in children with cerebral palsy: a preliminary study. Dev Med Child Neurol. 2002;44(7):447-60. http://dx.doi. org/10.1111/j.1469-8749.2002.tb00306.x

36. Bonomo LMM, Castro VC, Ferreira DM, Miyamoto ST. Hidroterapia na aquisição da funcionalidade de crianças com Paralisia Cerebral. Rev Neuroc. 2007;15(2):125-13.

37. Baleotti LR, Gritti CC, Silva BC. Efeitos de um protocolo modificado da terapia por contenção induzida em crianças com paralisia cerebral hemiparética. Rev Ter Ocup Univ São Paulo. 2014; 25(3):264-71. http://dx.doi.org/10.11606/issn.2238-6149.v25i3p264-271

38. Pavão S.L, Arnoni JLB, Oliveira AKC, Rocha NAC. Impacto de intervenção baseada em realidade virtual sobre o desempenho motor e equilíbrio de uma criança com paralisia cerebral: estudo de caso. Rev Paul Pediatr. 2014;32(4):389-94. http://dx.doi. org/10.1016/j.rpped.2014.04.005

39. Ferreira T, Pizzolatti ALA, Fontana HB, Polidoro BS, Silva CDC. Alongamento balístico versus sustentar e relaxar: comparação do ganho imediato e após 24 horas. Rev Fisioter Bras. 2010;11(2): 128-32.

40. Barato G, Fernandes T, Pacheco M, Bastos VH, Machado S, Mello MP, Silva JG, Orsini M. Plasticidade cortical e técnicas de fisioterapia neurológica na ótica da neuroimagem. Rev Neurocienc. 2008; 17(4):342-8.

41. Sanchez TC, Pio MRB. Abolição de zumbido evocado pela movimentação ocular por meio da repetição do deslocamento do olhar: um método inovador. Int Arch Otorhinolaryngol. 2007; 11(5):345-9.

42. Van Der NE, Verhage $\mathrm{CH}$, Groenendaal F, Vermeulen J, De Bode $\mathrm{S}$, Van Nieuwenhuizen O, De Vries LS. Neonatal neuroimaging predicts recruitment of contralesional corticospinal tracts following perinatal brain injury. Dev Med Child Neurol. 2013,55:707-12. http://dx.doi.org/10.1111/dmcn.12160

43. Holmström L, Vollmer B, Tedroff K, Islam M, Persson JK, Kits A, Forssberg $\mathrm{H}$, Eliasson AC. Hand function in relation to brain lesions and cortiço motor-projection pattern in children with unilateral cerebral palsy. Dev Med Child Neurol. 2010;52:145-52. http:// dx.doi.org/10.1111/j.1469-8749.2009.03496.x

44. Mackey A, Stinear C, Stott S, Byblow WD. Upper limb function and cortical organization in youth with unilateral cerebral palsy. Front Neurol. 2014;5(117):1-9. http://dx.doi.org/10.3389/ fneur.2014.00117

45. Islam M, Nordstrand L, Holmström L, Kits A, Forssberg H, Eliasson A. Is outcome of constraint-induced movement therapy in unilateral cerebral palsy dependent on corticomotor projection pattern and brain lesion characteristics? Dev Med Child Neurol. 2014;56:252-8. http://dx.doi.org/10.1111/dmcn.12353

46. Pihko E, Nevalainen P, Vaalto S, Laaksonen K, Mäenpää H, Valanne $\mathrm{L}$, Lauronen L. Reactivity of sensorimotor oscillations is altered in children with hemiplegic cerebral palsy: a magnetoencephalographic study. Hum Brain Mapp. 2014;35(8):4105-17. http://dx.doi. org/10.1002/hbm.22462

47. Koerte I, Pelavin P, Kirmess B, Fuchs T, Berweck S, Laubender RP, Borggraefe I, Schroeder S, Danek A, Rummeny C, Reiser M, Kubicki M, Shenton ME, Ertl-Wagner B, Heinen F. Anisotropy of transcallosal motor fibers indicates functional impairment in children with periventricular leukomalacia. Dev Med Child Neurol. 2011;53(2):179-6. http://dx.doi.org/10.1111/j.14698749.2010.03840.x

48. Kesar TM, Sawaki L, Burdette JH, Cabrera N, Kolaski K, Smith BP, O'Shea TM, Koman LA, Wittenberg GF. Motor Cortical Functional Geometry in Cerebral Palsy and its Relationship to Disability. Clin Neurophysiol. 2012;123(7):1383-90. http://dx.doi.org/10.1016/j. clinph.2011.11.005

49. Bleyenheuft Y, Dricot L, Gilis N, Kuo H, Grandin C, Bleyenheuft C, Gordon AM, Friel KM. Capturing neuroplastic changes after bimanual intensive. Res Dev Disabil. 2015;43-44:136-49. http:// dx.doi.org/10.1016/j.ridd.2015.06.014

50. Kirton, A. Can noninvasive brain stimulation measure and modulate developmental plasticity to improve function in stroke-induced cerebral palsy? Semin Pediatr Neurol. 2013;20(2):116-26. http:// dx.doi.org/10.1016/j.spen.2013.06.004

51. Boggio PS, Fregni F, Rigonatti SP, Marcolin MA, Silva MTA. Estimulação magnética transcraniana na neuropsicologia: novos horizontes em pesquisa sobre o cérebro. Rev Bras Psiquiatr. 2006;28(1):44-9. http://dx.doi.org/10.1590/S1516-44462006000100010 